\title{
Role of Conservative Management in Stab Penetrating Trauma to the Anterior Abdomen
}

\author{
Maher M Elzeiny, MD; Mohamed I Kassem, MD.
}

\author{
Department of Surgery, Gastrointestinal Surgery Unit, Faculty of medicine, \\ Alexandria University, Egypt
}

\begin{abstract}
Background/Purpose: Abdominal trauma is one of the major causes of death in trauma patients; abdominal trauma is the second leading cause of trauma deaths. This study was designed to evaluate the role of conservative management for selected cases of vitally stable patients who presented with stab penetrating trauma to the anterior abdomen with no evidence of peritonitis; hence avoiding unnecessary laparotomies aiming at reducing the hospital stay time and reducing morbidity and mortality.
\end{abstract}

Patients and methods: This prospective study was carried on 56 patients from May 2013 to October 2014. The work was done in the Emergency unit of the Alexandria Main University Hospital with stab penetrating trauma to the anterior abdomen. Patients were selected for Selective non operative management SNOM."

Results: 44 patients were males (78.57\%) and 12 patients were females (21.42\%). 24 patients (42.85\%) were less than 30 years, 22 patients (39.28\%) from 30-45 years and 10 patients (17.85\%) more than 45 years. The age ranged from 18 to 60 years with mean age of 33.3. Sixteen cases (28.5\%) had suspicious abdominal examination after admission. On exploration 11 cases were positive and 5 cases were negative. 34 cases (60.7\%) had free abdominal examination on admission, 26 cases were managed conservatively without complications, and hence they were saved unnecessary laparotomy while 8 cases were explored for other causes.

Conclusions: SNOM for abdominal stab wounds is no longer a novelty; its practice is now standard in most trauma centers. Vitally unstable cases with suspected internal haemorrhage should be immediately transferred to do exploratory laparotomy. It was concluded that SNOM of abdominal stab wounds is safe and preferred strategy for minimizing the days in hospital, hospital costs, as well as avoiding unnecessary laparotomies.

Key words: Stab wounds, Selective non operative management, Evisceration, Anterior abdomen.

Abbreviations: SNOM: Selective non operative management, FAST: Focused abdominal sonography for trauma

\section{Introduction:}

Abdominal trauma accounts for $8-10 \%$ of all trauma admissions to trauma centers; when unrecognized, abdominal trauma is one of the major causes of death in trauma patients. Abdominal trauma is the second leading cause of (preventable) trauma deaths. Increased incidences of deaths are secondary to hemorrhage and delay in receiving surgical intervention. ${ }^{1}$

Penetrating abdominal trauma can be life threatening because abdominal organs, especially those in the retroperitoneal space, can bleed profusely, and the space can hold a great deal of blood. If the pancreas is injured, it may be further injured by its own secretions, in a process called autodigestion. Injuries of the liver are common because of the size and location of the organ, present a serious risk for shock because the liver tissue is delicate and have a large blood supply and capacity. The intestines, taking a large part of the lower 
abdomen, are also at risk of perforation. 2,3,4

Stab and gunshot wounds account for most patient presentations of penetrating abdominal trauma, Stab wounds are encountered three times more often than gunshot wounds, but have a lower mortality because of their lower velocity and less invasive tract. As a result of their greater force and extensive missile tract, gunshot wounds account for up to $90 \%$ of the mortality associated with penetrating abdominal trauma. Injury to the bowel (small, then large) is most often found, followed by hepatic injury, regardless of type of penetrating injury. ${ }^{4}$ Stab wounds produce peritoneal violation in up to $70 \%$ of instances, but of these only one fourth to one third will require operative intervention. Stab wounds are classified in thirds. One third does not penetrate the peritoneal cavity, one third penetrates but do not damage and one third penetrate causing significant injury. 4,5

There are a large number of patients with penetrating abdominal trauma who have normal vital signs and negative abdominal examination when referred to trauma centers. A great deal of controversy exists between authorities about screening these patients for emergency laparotomy. Although the presence of classic indications such as shock, visceral evisceration and peritoneal irritation suggests laparotomy after penetrating abdominal stab wounds, the trend has moved in the past two decades from mandatory exploration to selective approach. ${ }^{1-6}$ Even in the presence of peritoneal violation, a significant number of patients have no major intra-abdominal injury requiring an operation. ${ }^{6}$ Mandatory laparotomy for penetrating abdominal stab wounds leads to unnecessary operations in $38-40 \%$ of patients, and postoperative morbidity ranges from $3 \%$ to $16 \% .{ }^{7,8}$

Many authors stressed that omental, intestinal evisceration, air underthe diaphragm and blood on abdominal paracentesis; none of these signs were an absolute criterion for mandatory exploration and that Selective Non Operative Management (SNOM) could be offered, even if with a heightened index of suspicion for injury and lowered threshold for an operation. ${ }^{9}$
It was concluded 9,10 that in the appropriate environment, SNOM of penetrating abdominal solid organ injuries has a high success rate and a low complication rate. The appropriate environment constituted a trauma center with a dedicated in-house team that could monitor and serially examine the patient during the observation period. In a prospective randomized study 11 with stab wounds to the anterior abdomen; were assigned to nonoperative management.

This study was designed to evaluate the role of conservative management for selected cases of vitally stable patients presented with penetrating trauma to the anterior abdomen with no evidence of peritonitis; hence avoiding unnecessary laparotomies aiming at reducing the hospital stay time and reducing morbidity and mortality.

\section{Patients:}

This work was conducted on 56 patients who were presented to the emergency unit of the Alexandria Main University Hospital with penetrating trauma to the anterior abdomen from May 2013 to October 2014. Patients were selected for SNOM according to the following criteria:

Inclusion criteria:

- Patients presenting with stab penetrating wounds to the anterior abdomen.

- Positive findings on local wound exploration (peritoneal violation).

Exclusion criteria:

- Vital instability on admission of the patient.

- Signs of peritonitis on admission of the patient.

After approval of local ethics committee, all patients included in the study were informed well about the management plan, possible benefits and side effects, and had an informed written consent.

\section{Methods:}

All patients in the present study were subjected to the following in order:

- Complete history taking; stressed on the mode of trauma, weapon used, time lapse 

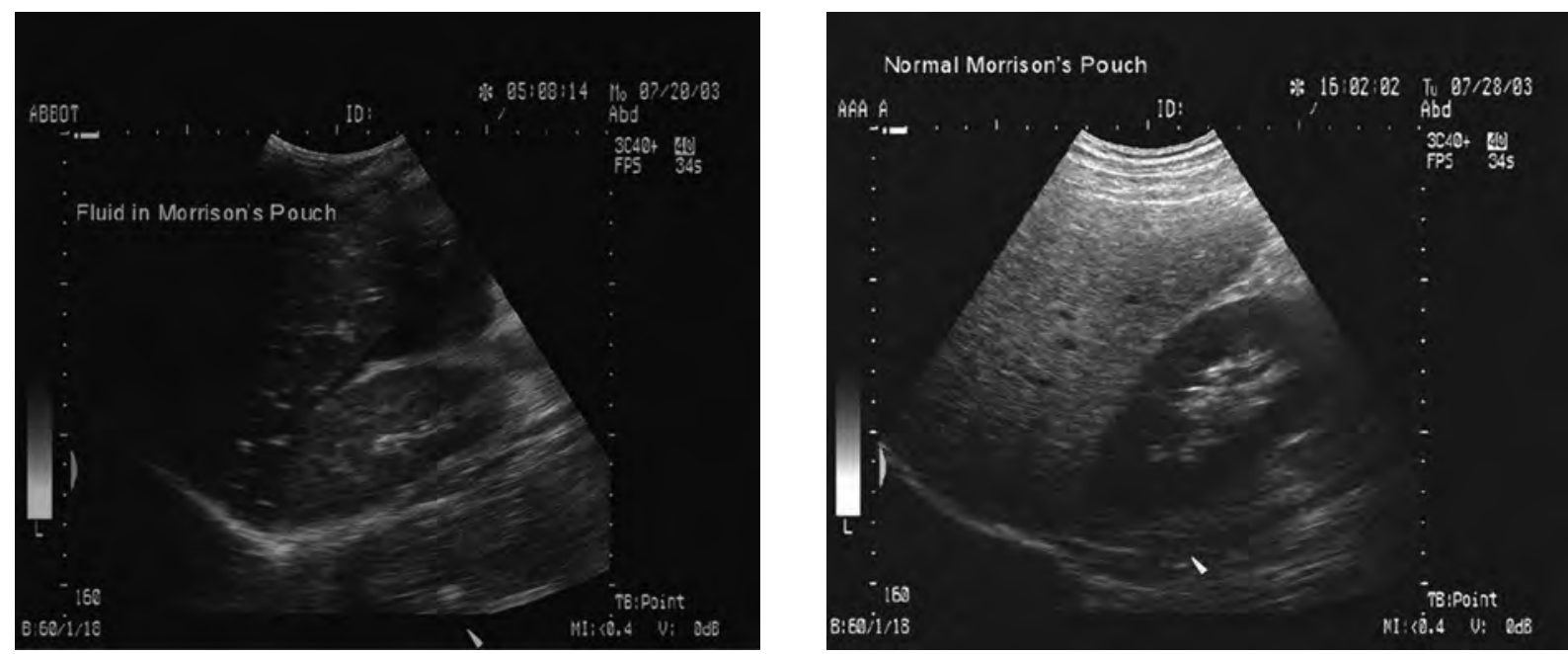

Figure (1): FAST can be a rapid method to identify intraperitoneal heamorrage.

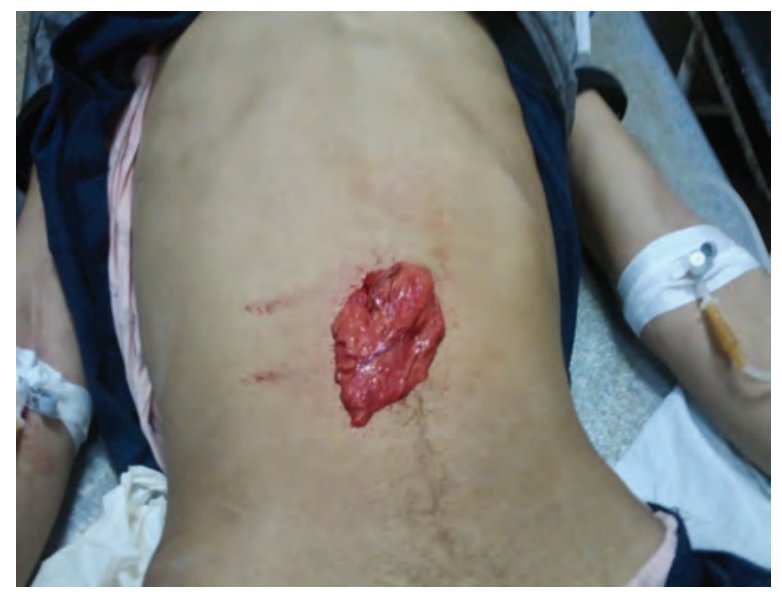

Figure (2): A 23 years old male patient presented to our ER ward by omentum evisceration at the umbilical region of anterior abdomen following stab wound.

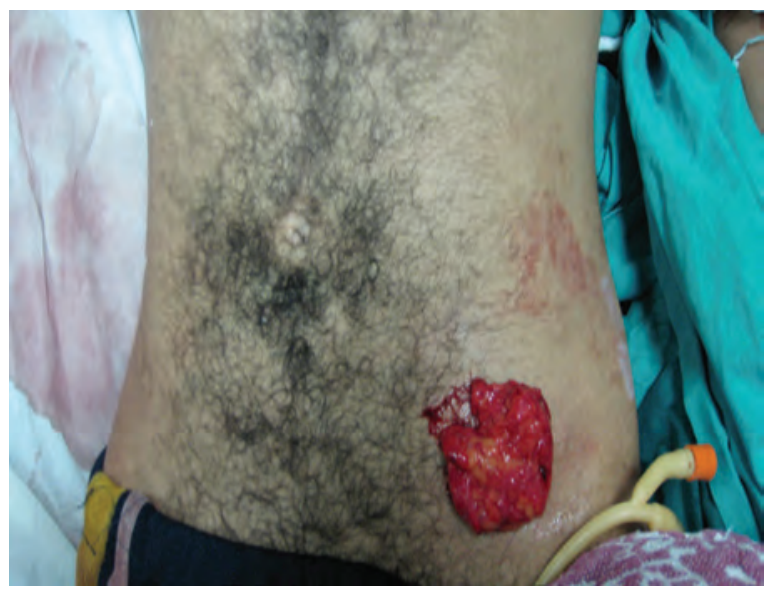

Figure (3): A 28 years old patient presented to our ER ward by evisceration of omentum at the left iliac fossa of anterior abdomen following a stab wound.

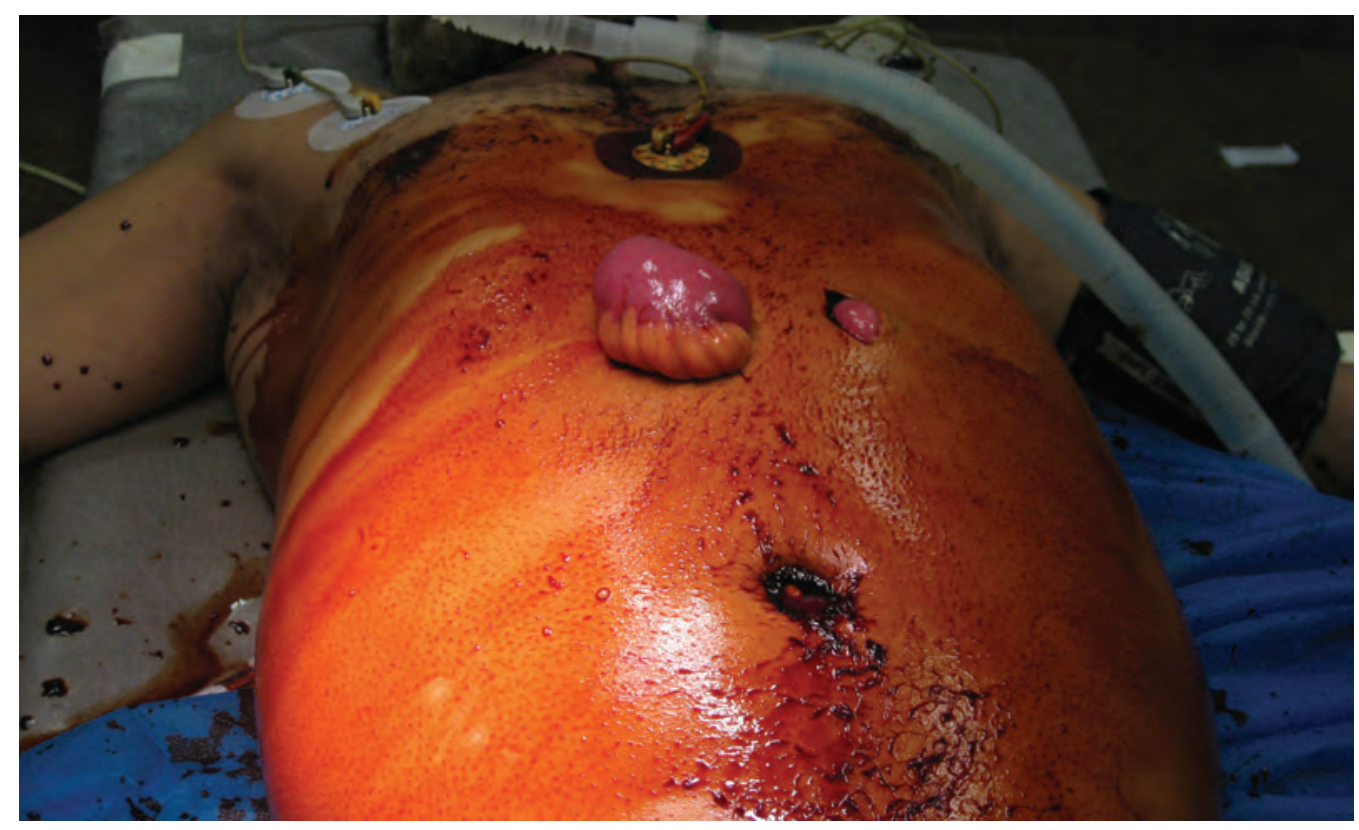

Figure (4): A 50 years old patient presented to our ER ward by 2 stab wounds showing evisceration of small bowel loops at the epigastric region of anterior abdomen. 
Table 1: The relation between vital stability and laparotomy on admission.

\begin{tabular}{|l|l|l|l|}
\hline & Laparotomy needed & Laparotomy not Needed & Total \\
\hline Vitally stable & 16 & 34 & 50 \\
\hline Vitally unstable & 6 & 0 & 6 \\
\hline
\end{tabular}

Table 2: The relation between findings of abdominal examination and the need for laparotomy.

\begin{tabular}{|c|c|c|c|c|c|}
\hline \multirow{2}{*}{\multicolumn{3}{|c|}{ Characteristics: }} & \multicolumn{2}{|c|}{ Was laparotomy Needed? } & \multirow{2}{*}{ Total } \\
\hline & & & No & Yes & \\
\hline \multirow{4}{*}{$\begin{array}{l}\text { Abdominal } \\
\text { examination: }\end{array}$} & \multirow[t]{2}{*}{ Free } & Count & 26 & 8 & 34 \\
\hline & & $\%$ & $46.42 \%$ & $14.28 \%$ & $60.7 \%$ \\
\hline & \multirow[t]{2}{*}{ Suspicious } & Count & 5 & 11 & 16 \\
\hline & & $\%$ & $8.33 \%$ & $19.6 \%$ & $28.5 \%$ \\
\hline & \multirow[t]{2}{*}{ Total } & Count & 31 & 19 & 50 \\
\hline & & $\%$ & $55.35 \%$ & $33.92 \%$ & $89.28 \%$ \\
\hline
\end{tabular}

Table 3. U/S categories of free I. P. Collection and significant laparotomy.

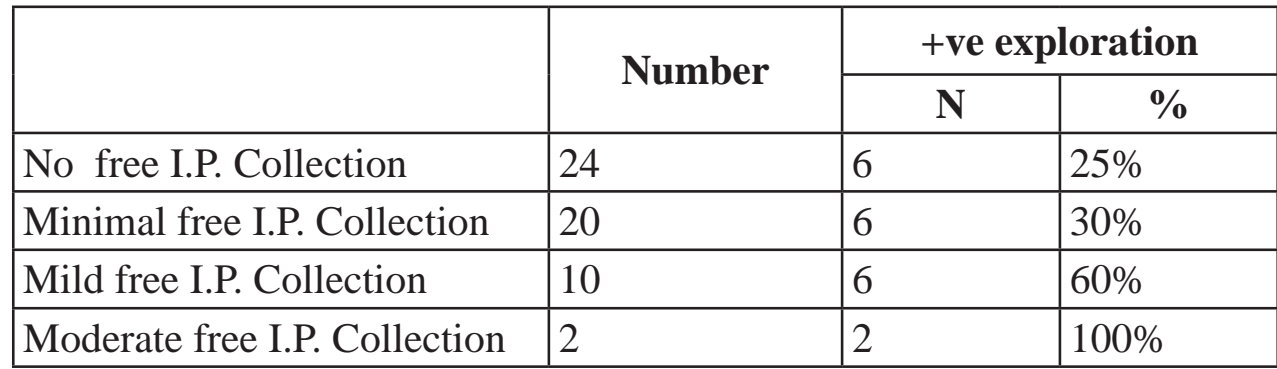

I.P. Collection = intraperitoneal collection (equivalent to McKenney scores).

between the trauma and hospital admission.

- Thorough serial physical examination at admission including:

- Clinical :

- Vital signs :

- Arterial blood pressure, pulse.

- Temperature: Basal temperature of the patient was recorded.

- Local abdominal examination :

- Complete abdominal examination; stressed on signs of peritonitis (hyperaesthesia of the skin, superficial tenderness, rebound tenderness and board like rigidity).

Patients who would not undergo laparotomy on the basis of the previous measures were subjected to the following:

- Lab. :

- Complete blood count: Rise of white blood count indicated peritonitis whereas fall of heamoglobin or heamatocrit indicated ongoing process of bleeding.

- Imaging:

- Ultrasonography of the abdomen; Focused Abdominal Sonography for Trauma (FAST). To confidently exclude significant intraperitoneal injury in patients with negative FAST scan findings and for patients with positive FAST findings additional investigations were required (Fig. 1).

- According to the protocols applied by Diagnostic Radiology department of Alexandria Main University Hospital ; the results of FAST was categorized into 4 main categories :

- Free (no free intraperitoneal fluid collection).

- Minimal collection (equivalent to McKenney score 1) 
- Mild collection. (equivalent to McKenney score 2)

- Moderate collection. (equivalent to McKenney score 3) \{This correlation was applied in 2004 in a thesis submitted to the radiology department, Alexandria University, McKenney et al ${ }^{12,13}$ evaluated a scoring system that measured the depth of fluid in the deepest pocket, and 1 point was added for fluid in each of the other areas (four areas maximum)\}.

- CT scan: ( plain cuts in cases with negative FAST findings and with IV contrast in cases with positive FAST to identify the source of bleeding):

- Evidence of pneumoperitoneum was considered as an indication of laparotomy.

- Evident solid organ injury, (liver or spleen), with active contrast extravasation was an indication of laparotomy.

- Local wound exploration :

- Local wound exploration was done under local anaesthesia.

- If omentum was protruding through the wound, it was returned into the peritoneal cavity.

- Evisceration of organs - other than omentum - was considered an indication for immediate laparotomy.

Patients who would not undergo laparotomy on the basis of the previous measures were selected for "selective non operative management SNOM" and were subjected to the following:

Thorough serial physical examination every 4 hours for the first 24 hours; then every 6 hours for the next 24 hours; including vital signs and abdominal examination.

\section{Follow up:}

Patients who were managed conservatively were followed up for 3 months after discharge by clinical re-evaluation and ultrasonography and multislice CT abdomen and pelvis if needed.

\section{Results:}

Age and gender: 44 patients were males (78.57\%) where 12 patients were females (21.42\%). 24 patients (42.85\%) were less than 30 years, 22 patients (39.28\%) from 30 45 years and 10 patients (17.85\%) more than 45 years. The age ranged from 18 to 60 years with mean age of 33.3.

Mode of trauma: The fifty six patients presented by stab wounds ( 4 of them had 2 stab wounds).

Site of trauma: Eighteen patients (30\%) were stabbed in the epigastrium, 6 patients $(10 \%)$ in the umbilical region, 5 patients (8.9\%) in the right hypochondrium, 6 patients $(10 \%)$ in the left hypochondrium, 7 patients $(11.67 \%)$ in the left lumbar, 2 patients (33.34\%) in the right lumbar region, 6 patients (10.7\%) in the right iliac fossa and 6 patients (10\%) in the left iliac fossa. Nine cases $(18.34 \%)$ presented by evisceration $\{7(11.67 \%)$ cases presented with omentum evisceration, $1(1.7 \%)$ case presented with evisceration of the small intestine and the last case (1.7\%) presented by colon evisceration Figures $(2,3,4)$.

On admission: Out of 56 patients, 6 patients (10.7\%) were vitally unstable with suspected internal haemorrhage after about 4 hours from admission during close follow up period. They were immediately transferred to do exploratory laparotomy. The six explorations were positive Table (1).

- One of these cases was injured by two stab wounds in the right hypochondrium and umbilical region, exploration revealed injury to the stomach, Duodenum (11 ${ }^{\text {st }}$ part), transverse colon and small intestine.

- In the other 5 cases: The small intestine was injured in 2 cases but the colon, liver and spleen each injured in 1 case.

Therefore according to our results, vital instability was a good indicator to the actual need for laparotomy, whereas vital stability did not exclude the need for laparotomy.

The remaining 50 patients (89.28\%) were evaluated using 3 main tools:

-Abdominal examination.

- Ultrasonography.

- CT scan.

Abdominal examination: Table (2)

In this study abdominal examination was considered as an indication for laparotomy regardless of other investigations: 
- 16 cases (28.5\%) had suspicious abdominal examination after admission during close follow up (within 24 hours from admission), on exploration 11 cases (19.6\%) were positive and 5 cases (8.33\%) were negative.

- 34 cases $(60.71 \%)$ had free abdominal examination on admission, 26 cases (46.42\%) of them passed conservative without complications, hence were saved from unnecessary laparotomy while 8 cases (14.28\%) were explored for other causes:

- 4 cases had-ve explorations:

- 3 cases had delayed explorations; 24 hours after admission due to vital instability and increase in the free intraperitoneal collection by U/S, the source of bleeding was parietal from the anterior abdominal wall muscles.

- One case was explored due to pneumoperitoneum on CT scan.

- 4 cases had +ve explorations:

- 2 cases were explored due to active contrast extravasations on the basis of CT scan, one case from the liver (required surgical repair), the other case from the spleen (required splenectomy).

- 2 cases were explored; 36 hours after admission due to vital instability and increase in the free intraperitoneal collection by U/S, small intestinal perforations were found with bleeding from the mesentery.

Hence abdominal examination could detect the need for laparotomy with the following sensitivity and specificity:

- Sensitivity: 76.9\%.

- Specificity: $81.8 \%$.

\section{Ultrasonography:}

50 cases were evaluated using U/S as 6 cases were vitally unstable and were explored without any further investigations Table (3).

The table showed that only patients with major free intraperitoneal collection could be operated without further investigations.

\section{CT scan:}

46 patients were evaluated using multislice CT abdomen and pelvis (for detection of solid organ injury, collection, pneumoperitoneum, and follow up) were:

6 cases (10\%) had free intraperitoneal air in CT scan (pneumoperitoneum):

- 5 cases had +ve explorations.

- 1 case had -ve exploration.

This study concluded that free intraperitoneal air in CT plus suspicious abdominal examination necessitated laparotomy.

- 2 cases $(3.33 \%)$ were explored on CT basis due active contrast extravasation.

\section{Evisceration:}

- 7 cases $(11.67 \%)$ presented with omentum evisceration and none had pneumoperitoneum on CT basis. 4 cases were explored on the basis of suspicious abdominal examination:

- 3 cases showed -ve explorations.

- 1 case showed splenic laceration that required laparotomy.

- 2 cases presented with evisceration of the intestine (1 small intestine and the other was the colon). Both showed perforation on explorations.

Selective Conservative Management:

34 cases (60.71\%) were selected for conservative management. 26 cases $(46.42 \%)$ of them passed conservative without complications, so saved from unnecessary laparotomy.

Only 8 cases out of 34 needed delayed laparotomy was done:

- 3 cases had bleeding from the anterior abdominal wall muscles.

- One case was explored due to pneumoperitoneum on CT scan.

- 2 cases were explored due to active contrast extravasations on the basis of CT scan, one case from the liver, the other case from the spleen.

- 2 cases were explored due to vital instability and increase in the free intraperitoneal collection from small intestinal perforation and bleeding from the mesentery.

\section{Discussion:}

Leppaniemi et $\mathrm{al}^{14}$ conducted a retrospective analysis of hospital records of 
209 patients with anterior abdominal stab wounds over 4 years, 175 cases were males, 34 females, and mean age 36 years. Lee et $\mathrm{al}^{15}$ conducted a retrospective study by reviewing the medical records of 219 patients with stab wounds to the anterior abdomen over the period of 10 years, the age ranged from 9 years to 64 years, mean age 26.6 years. 89.5 $\%$ of the cases were males, and only $10.5 \%$ were females.

This present study was conducted over one and half years on patients with penetrating wounds to the anterior abdominal wall, out of 56 patients, 44 patients were males $(78.57 \%)$ where 12 patients were females (21.42\%). The age ranged from 18 to 60 years with mean age of 33.3. The fifty six patients presented by stab wounds (4 of them had 2 stab wounds).

Certain studies ${ }^{14-16}$ stated that unexplained shock is highly accurate as an indicator of intra-abdominal injury and the presence of shock was a mandatory indication for early exploration; and this is conformed to the present study. On the contrary, other studies ${ }^{9}$ denied shock at admission as an indication for early exploration and conservative management was successfully offered without complications and with no mortality.

Results obtained by Demetriades et $\mathrm{al}^{9}$ were more accurate; 311 cases had had normal abdominal findings on examination; 302 cases proved not to have intra-abdominal injuries requiring exploration, only 9 cases needed therapeutic laparotomies; 340 cases had suspicious abdominal examination; 329 cases needed therapeutic laparotomies, while 11 cases proved not to have intra-abdominal injuries requiring exploration (sensitivity: 97.3\%, specificity: 96.4\%).

Leppaniemi et al ${ }^{14}$ conducted a study which confirmed that early laparotomy based on signs of generalized peritonitis associated with a relatively low risk of a negative operation. Out of 36 patients presented with generalized abdominal tenderness on admission only seven patients had no intraabdominal injuries requiring surgical repair. Out of 57 patients with a gastrointestinal tract perforation, 9 underwent immediate laparotomy for haemodynamic instability,
26 for generalized abdominal tenderness, whereas 14 patients showed no initial signs of generalized tenderness or other positive findings. In a study conducted by McKinney et al,12,13 $85 \%$ of patients with a score $\geq 3$ required a therapeutic laparotomy, whereas $15 \%$ of patients with a score of $<2$ required surgery. Boulanger $\mathrm{BR}$ et $\mathrm{al}^{17}$ stated that the routine use of sonography in penetrating torso injury was beneficial. The detection of peritoneal fluid was clinically useful. However, a negative FAST examination did not exclude abdominal injury, such as a diaphragm or hollow viscus wound, and further investigation or close follow-up was required. The results in the literatures ${ }^{17-20}$ conformed to the results in our study.

Munera $\mathrm{F}$ et $\mathrm{al}^{21}$ stated that in stable patients with gunshot wounds to the abdomen in whom there was no indication for immediate surgery, triple-contrast helical CT could help reduce the number of cases of unnecessary or nontherapeutic laparotomy (negative laparotomy) and could help identify patients with injuries that might be safely treated without surgery. Chiu WC et $\mathrm{al}^{22}$ reported that CT accurately predicted whether laparotomy was needed in 71/75 (95\%) patients. In patients with negative CT, 47/49 (96\%) had successful nonoperative management and 1 had negative laparotomy. The single CT-missed peritoneal violation had a left diaphragm injury at laparotomy.

In the present study, CT scan was used as a useful tool of diagnosing solid organ injuries and hollow viscus perforations namely the stomach, but showed no role for diagnosis of small intestinal perforation. "Pneumoperitoneum" by CT scan was considered as an absolute indication of laparotomy.

Organ evisceration was mostly accepted as an indication for emergency laparotomy. The need for surgical intervention reached a rate of $70 \%-80 \%$ in cases with penetrating abdominal stab injuries with organ evisceration. ${ }^{23-28}$ While 2 out of 3 patients with organ evisceration requiring surgical intervention had an intra-abdominal injury, 1 had no such injury. Omental evisceration, 
on the other hand, was not an indication for routine laparotomy. ${ }^{9,24,25}$ The omentum was irrigated, cleaned, and reduced to the abdominal cavity. Risk of intra-abdominal injury was higher in such patients compared to those with no evisceration. Laparotomy was therapeutic in 9 of 12 (75\%) when performed for a penetrating abdominal stab injury without evisceration, while laparotomy was therapeutic in 12 out of 13 patients (92\%) undergoing laparotomy for evisceration. ${ }^{26}$

The policy of mandatory laparotomy led to many negative laparotomies. In some series up to $53 \% .{ }^{29}$ Although some authors reported no mortality in the negative laparotomy but others could not achieve this results (Mayards found $6.3 \%$ mortality rate, ${ }^{30}$ a negative laparotomy performed as an emergency without any patient preparation, poor anesthetics facilities; untrained anesthetists posed a significant risk. Most patients had full stomach and were prone to aspiration during induction of anesthesia. Nance et al ${ }^{16}$ stated that the policy of selective observation for 393 stab wounds has reduced the percentage of negative explorations from $53 \%$ to $11 \%$ and reduced the percentage of patients subjected to exploration from $95 \%$ to $45 \%$.

\section{Conclusion and recommendations:}

1- Vitally unstable cases with suspected internal haemorrhage should immediately transferred to do exploratory laparotomy without any further investigations.

2- Local abdominal examination is the most reliable method for detection of significant intra-abdominal injuries requiring laparotomy and should never be replaced by medical imaging namely US and CT.

3- Serial clinical evaluation is essential for abdominal stab wound management. Close monitoring and follow-up are mandatory in patients managed non-operatively. Repeated clinical examination - by preferably the same physician - over the 12-24 hours following arrival to the hospital is necessary.

4- Only patients who have massive internal hemorrhage detected by US can undergo laparotomy without further investigations.
5- CT scan can accurately detect internal hemorrhage and significant solid organ injuries requiring laparotomy but caution must still be maintained in diagnosing individual hollow viscus injuries by CT.

6- Evisceration of omentum following abdominal stab wounds should not be an absolute indication for early laparotomy while evisceration of colon or small intestine should be an indication for early laparotomy without further investigations.

7- It was concluded that SNOM of abdominal stab wounds, although resulting in delayed laparotomy in some patients, is safe and the preferred strategy for minimizing; the days in the hospital, hospital costs as well as avoiding unnecessary laparotomies.

\section{Reference:}

1- Butt MU, Zacharias N, Velmahos GC: Penetrating abdominal injuries: Management controversies. Scand J Trauma Emerg Med 2009; 17-19.

2- Navsaria PH, Berli JU, Edu S, Nicol AJ: Non-operative management of abdominal stab wounds - an analysis of 186 patients. $S$ Afr J Surg 2007; 45: 128-132.

3- Demetriades D, Rabinowitz B, Sofianos C: Non-operative management of penetrating liver injuries: A prospective study. Br J Surg 1986; 73: 736-737.

4- Nicholas JM, Rix EP, Easley KA, et al: Changing patterns in the management of penetrating abdominal trauma: The more things change, the more they stay the same. J Trauma 2003; 55: 1095-1110.

5- Da Silva M, Navsaria PH, Edu S, Nicol AJ: Evisceration following abdominal stab wounds: Analysis of 66 cases. World J Surg 2009; 33(2): 215-219.

6- Nair MS, Uzzaman MM, Al-Zuhir N, Jadeja A, Navaratnam R: Changing trends in the pattern and outcome of stab injuries at a North London hospital. J Emerg Trauma Shock 2011; 4(4): 455-460.

7- Demetriades D, Hadjizacharia P, Constantinou $\mathrm{C}$, Brown $\mathrm{C}$, Inaba $\mathrm{K}$, Rhee $\mathrm{P}$, Salim A: Selective nonoperative management of penetrating abdominal solid organ injuries. Ann Surg 2006; 244: 620-628.

8- Inaba K, Demetriades D: The nonoperative management of penetrating abdominal trauma. Adv Surg 2007; 41: 51-62.

9- Demetriades D, Rabinowitz B: Indications 
for operation in abdominal stab wounds. A prospective study of 651 patients. Ann Surg 1987; 205: 129-132.

10- Arikan S, Kocakusak A, Yucel AF, Adas G: A prospective comparison of the selective observation and routine exploration methods for penetrating abdominal stab wounds with organ or omentum evisceration. J Trauma 2005; 58(3): 526-532.

11- Leppäniemi AK, Haapiainen RK: Selective nonoperative management of abdominal stab wounds: Prospective, randomized study. World J Surg 1996; 20: 1101-1109.

12- McKenney KL, McKenney MG, Cohn SM, et al: Hemoperitoneum score helps determine need for therapeutic laparotomy. J Trauma 2001; 50: 650-654.

13- Ong AW, McKenney MG, McKenney KA, et al: Predicting the need for laparotomy in pediatric trauma patients on the basis of the ultrasound score. J Trauma 2003; 54: 503-508.

14- Leppaniemi AK, Voutilainen PE, Haapiainen $\mathrm{RK}$, et al: Indications for early mandatory laparotomy in abdominal stab wounds. $\mathrm{Br} \mathrm{J}$ Surg 1999; 86: 76-80.

15- Lee WC, Uddo JF, Nance FC, et al: Surgical judgment in the management of abdominal stab wounds utilizing clinical criteria from a 10-year Experience. Ann Surg 1984; 199: 549-553.

16- Nance FC, Wennar MH, Johnson LW, et al: Surgical judgment in the management of penetrating wounds of the abdomen: Experience with 2,212 patients. Ann Surg 1974; 179: 639-646.

17- Boulanger BR, Kearney PA, Tsuei B, Ochoa JB: The routine use of sonography in penetrating torso injury is beneficial. $J$ Trauma 2001; 51(2): 320-325.

18- Udobi KF, Rodriguez A, Chiu WC, Scalea TM: Role of ultrasonography in penetrating abdominal trauma: A prospective clinical study. J Trauma 2001; 50(3): 475-479.

19- Quinn AC, Sinert R: What is the utility of the Focused Assessment with Sonography in Trauma (FAST) exam in penetrating torso trauma? Injury 2011; 42(5): 482-487.

20- Tayal VS, Beatty MA, Marx JA, Tomaszewski CA, Thomason MH: FAST (focused assessment with sonography in trauma) accurate for cardiac and intraperitoneal injury in penetrating anterior chest trauma. $J$ Ultrasound Med 2004; 23(4): 467^472.

21- Múnera F, Morales C, Soto JA, Garcia HI, Suarez T, Garcia V, Corrales M, Velez G: Gunshot wounds of abdomen: Evaluation of stable patients with triple-contrast helical CT. Radiology 2004; 231(2): 399-405.

22- Chiu WC, Shanmuganathan K, Mirvis SE, Scalea TM: Determining the need for laparotomy in penetrating torso trauma: A prospective study using triple-contrast enhanced abdominopelvic computed tomography. J Trauma 2001; 51(5): 860-868.

23- Schmelzer TM, Mostafa G, Gunter OL Jr, Norton HJ, Sing RF: Evaluation of selective treatment of penetrating abdominal trauma. $J$ Surg Educ 2008; 65: 340-345.

24- Ertekin C, Yanar H, Taviloglu K, Güloglu $\mathrm{R}$, Alimoglu O: Unnecessary laparotomy by using physical examination and different diagnostic modalities for penetrating abdominal stab wounds. Emerg Med J 2005; 22: 790-794.

25- Huzinga WK, Baker LW, Mtshali ZW: Selective management of abdominal and thoracic stab wounds with established peritoneal penetration: The eviscerated omentum. Am J Surg 1987; 153: 564-568.

26- Yucel M, Caliskan M, Sisik A, Subası İE, Ozpek A, Basak F, et al: Penetrating anterior abdominal stab injury. DEU Faculty of Medicine 2011; 25: 175-180.

27- Silva M, Navsaria PH, Edu S, Nicol AJ: Evisceration following abdominal stab wounds: Analysis of 66 cases. World J Surg 2009; 33: 215-219.

28- Medina M, Ivatury RR, Stahl WM: Omental evisceration through an abdominal stab wound: Is exploratory laparotomy mandatory. Can J Surg 1984; 4: 399-401.

29- Forde K, Ganepola G: Is mandatory exploration for penetrating abdominal trauma extinct. The morbidity and mortality of negative exploration in large municipal hospital. J Truma 1974; 14: 764-766.

30- Mayard A, Oropeza G: Mandatory operation for penetrating wounds of abdomen. Am J Surg 1968; 115: 307-312. 